Pacific

Journal of

Mathematics

\title{
GENUS-TWO GOERITZ GROUPS \\ OF LENS SPACES
}

SANGBUM ChO

Volume 265 No. 1

September 2013 


\title{
GENUS-TWO GOERITZ GROUPS OF LENS SPACES
}

\author{
SANGBUM CHO
}

\begin{abstract}
Given a genus-g Heegaard splitting of a 3-manifold, the Goeritz group is defined to be the group of isotopy classes of orientation-preserving homeomorphisms of the manifold that preserve the splitting. In this work, we show that the Goeritz groups of genus-2 Heegaard splittings for lens spaces $L(p, 1)$ are finitely presented, and give explicit presentations of them.
\end{abstract}

\section{Introduction}

It is well known that every closed orientable 3-manifold $M$ can be decomposed into two handlebodies of the same genus. This is what we call a Heegaard splitting of the manifold, and the genus of the handlebodies is called the genus of the splitting. Given a genus- $g$ Heegaard splitting of $M$, the Goeritz group of the splitting, which we will denote by $\mathscr{G}_{g}$, is the group of isotopy classes of orientation-preserving homeomorphisms of $M$ that preserve each of the handlebodies of the splitting setwise. In particular, this group is interesting when the manifold is the 3-sphere or a lens space since it is well known from [Waldhausen 1968; Bonahon 1983; Bonahon and Otal 1983] that they have unique Heegaard splittings for each genus up to isotopy. In this case, each Goeritz group depends only on the genus of the splitting, and so we can define the genus-g Goeritz group $\mathscr{G}_{g}$ of each of those manifolds without mentioning a specific Heegaard splitting. For the 3-sphere, it was shown in [Goeritz 1933; Scharlemann 2004] that $\mathscr{G}_{2}$ is finitely generated, and subsequently in [Akbas 2008; Cho 2008] that $\mathscr{G}_{2}$ is finitely presented and its finite presentation was introduced. Further, in [Koda 2011], a natural generalization of a Goeritz group is studied, namely, the group of isotopy classes of orientationpreserving homeomorphisms of the 3-sphere preserving an embedded genus-two handlebody which is possibly knotted.

This work is supported by the Basic Science Research Program through the National Research Foundation of Korea (NRF) and funded by the Ministry of Education, Science, and Technology (2012006520).

MSC2010: primary 57N10; secondary 57M60.

Keywords: Heegaard splitting, Goeritz group, lens space, disk complex. 
In this work, we show that the Goeritz group $\mathscr{G}_{2}$ of each of the lens spaces $L(p, 1)$ is finitely presented. In the main theorem, Theorem 5.4, their explicit presentations are given. For the genus-2 Goeritz groups of the other lens spaces, and for the higher genus Goeritz groups of the 3-sphere and lens spaces, it is conjectured that they are all finitely presented, but it is still known to be an open problem.

We generalize the method developed in [Cho 2008]. We find a tree on which $\mathscr{G}_{2}$ for $L(p, 1)$ acts such that the quotient of the tree by the action of $\varphi_{2}$ is a single edge, and then apply the well known theory of groups acting on trees due to Bass and Serre (see [Serre 1980]). Such a tree will be found in the barycentric subdivision of the disk complex for one of the handlebodies of the splitting. For arbitrary lens spaces $L(p, q)$, finding such trees, if they exist, is a much more complicated problem than $L(p, 1)$, which will be fully discussed in [Cho and Koda 2012].

Throughout the paper, we simply denote by $\mathscr{G}$ the genus-2 Goeritz group $\mathscr{G}_{2}$ of a lens space. We use the standard notation $L(p, q)$ with $p \geq 2$ for a lens space with its basic properties found in standard textbooks. For an example, we refer to [Rolfsen 1976]. For a genus-1 Heegaard splitting of $L(p, 1)$, any oriented meridian circle of a solid torus of the splitting is identified with a $(p, 1)$-curve (or a $(p, p-1)$-curve) on the boundary of the other solid torus after a suitable choice of oriented longitude and meridian of the other solid torus is made. The triple $(V, W ; \Sigma)$ will denote a genus-2 Heegaard splitting of a lens space $L=L(p, q)$. That is, $L=V \cup W$ and $V \cap W=\partial V=\partial W=\Sigma$, where $V$ and $W$ are handlebodies of genus two.

The disks $D$ and $E$ in a handlebody are always assumed to be properly embedded, and their intersection is transverse and minimal up to isotopy. In particular, if $D$ intersects $E$, then $D \cap E$ is a collection of pairwise disjoint arcs that are properly embedded in both $D$ and $E$. Finally, $\operatorname{Nbd}(X)$ will denote a regular neighborhood of $X$, and $\operatorname{cl}(X)$ the closure of $X$ for a subspace $X$ of a polyhedral space where the ambient space will always be clear from the context.

\section{Primitive elements of the free group of rank two}

The fundamental group of the genus- 2 handlebody is the free group $\mathbb{Z} * \mathbb{Z}$ of rank two. We call an element of $\mathbb{Z} * \mathbb{Z}$ primitive if it is a member of a generating pair of $\mathbb{Z} * \mathbb{Z}$. Primitive elements of $\mathbb{Z} * \mathbb{Z}$ have been well understood. For an example we refer [Osborne and Zieschang 1981] to the reader. A key property of the primitive elements of the free group of rank two is the following, which is a direct consequence of Corollary 3.3 in [Osborne and Zieschang 1981]:

Proposition 2.1. Fix a generating pair $\{x, y\}$ of $\mathbb{Z} * \mathbb{Z}$, and let $w$ be a primitive element of $\mathbb{Z} * \mathbb{Z}$. Then for some $\epsilon \in\{1,-1\}$ and some $n \in \mathbb{Z}$, some cyclically reduced form of $w$ is a product of terms of the form $x^{\epsilon} y^{n}$ or $x^{\epsilon} y^{n+1}$, or else a product of terms of the form $y^{\epsilon} x^{n}$ or $y^{\epsilon} x^{n+1}$. 
From the proposition, the cyclically reduced forms of a primitive element are very restrictive. For example, if $w$ is a primitive element of $\mathbb{Z} * \mathbb{Z}$, then no cyclically reduced form of $w$ in terms of $x$ and $y$ can contain $x$ and $x^{-1}$ (and $y$ and $y^{-1}$ ) simultaneously.

A simple closed curve in the boundary of a genus- 2 handlebody $W$ represents an element of $\pi_{1}(W)=\mathbb{Z} * \mathbb{Z}$. We call a pair of essential disks in $W$ a complete meridian system for $W$ if the union of the two disks cuts up $W$ into a 3-ball. Given a complete meridian system $\{F, G\}$, assign symbols $x$ and $y$ to circles $\partial F$ and $\partial G$ respectively. Suppose that an oriented simple closed curve $l$ on $\partial W$ meets $\partial F \cup \partial G$ transversely and minimally. Then $l$ determines a word in terms of $x$ and $y$ which can be read off from the intersections of $l$ with $\partial F$ and $\partial G$ (after a choice of orientations of $\partial F$ and $\partial G)$, and hence $l$ represents an element of the free group $\pi_{1}(W)=\langle x, y\rangle$.

In this set up, the following is a simple criterion for the primitiveness of the elements represented by such a simple closed curve:

Lemma 2.2. With a suitable choice of orientations of $\partial F$ and $\partial G$, if a word determined by the simple closed curve l contains one of the subwords $y x y^{-1}$ or $x y x y^{n}$ for $n \geq 3$, then any element in $\pi_{1}(W)$ represented by $l$ cannot be a primitive element.

Proof. Let $\Sigma^{\prime}$ be the 4-holed sphere cut up from $\partial W$ along $\partial F \cup \partial G$, and denote by $f_{+}$and $f_{-}$(respectively $g_{+}$and $g_{-}$) the boundary circles of $\Sigma^{\prime}$ that came from $\partial F$ (respectively $\partial G)$.

Suppose first that a word represented by $l$ contains a subword of the form $y x y^{-1}$. Then we may assume that there are two arcs $l_{+}$and $l_{-}$of $l \cap \Sigma^{\prime}$ such that $l_{+}$connects $f_{+}$and $g_{+}$, and $l_{-}$connects $f_{+}$and $g_{-}$as in Figure 1, left. Since $\left|l \cap f_{+}\right|=\left|l \cap f_{-}\right|$ and $\left|l \cap g_{+}\right|=\left|l \cap g_{-}\right|$, we must have two other arcs $m_{+}$and $m_{-}$of $l \cap \Sigma^{\prime}$ such that $m_{+}$connects $f_{-}$and $g_{+}$, and $m_{-}$connects $f_{-}$and $g_{-}$. We see then that there exists no arc component of $l \cap \Sigma^{\prime}$ that meets only one of $f_{+}, f_{-}, g_{+}$or $g_{-}$. That is, any word determined by $l$ contains neither $x^{ \pm 1} x^{\mp 1}$ nor $y^{ \pm 1} y^{\mp 1}$, and so each word is cyclically reduced, but a word determined by $l$ already contains both $y$ and $y^{-1}$, and so $l$ cannot represent a primitive element of $\pi_{1}(W)$ by Proposition 2.1.

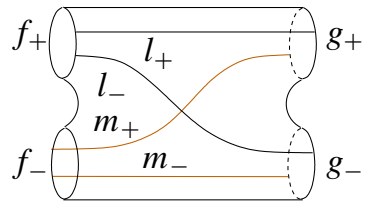

$\Sigma^{\prime}$

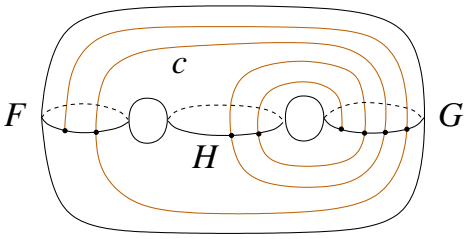

W

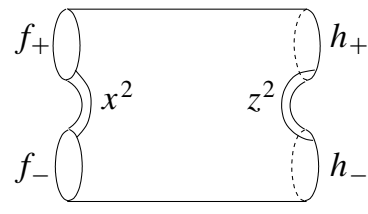

$\Sigma^{\prime \prime}$

Figure 1. The 4-holed spheres $\Sigma^{\prime}$ and $\Sigma^{\prime \prime}$. 
Next, suppose that a word represented by $l$ contains a subword of the form $x y x y^{n}$ for $n \geq 3$. We may assume there is an arc $c$ of $l \cap \Sigma^{\prime}$ connecting $f_{+}$and $g_{+}$in $\Sigma^{\prime}$. Consider the circle which is the frontier of a regular neighborhood of $f_{+} \cup c \cup g_{+}$ in $\Sigma^{\prime}$. This circle bounds a disk $H$ in $W$, and $\{F, H\}$ forms a complete meridian system of $W$. Assigning symbols $x$ and $z$ to $\partial F$ and $\partial H$ respectively, the circle $l$ represents an element of $\pi_{1}(W)=\langle x, z\rangle$ (see Figure 1, middle).

Let $\Sigma^{\prime \prime}$ be the 4-holed sphere cut up from $\partial W$ along $\partial F \cup \partial H$, and denote by $f_{+}$and $f_{-}$(respectively $h_{+}$and $h_{-}$) the boundary circles of $\Sigma^{\prime \prime}$ that came from $\partial F$ (respectively $\partial H$ ). There are two arcs of $l \cap \Sigma^{\prime \prime}$ such that one connects $f_{+}$and $f_{-}$, and the other one connects $h_{+}$and $h_{-}$. We may assume that these two arcs represent subwords of the form $x^{2}$ and $z^{2}$ (see Figure 1, right). Thus there exists no arc component of $l \cap \Sigma^{\prime \prime}$ that meets only one of $f_{+}, f_{-}, h_{+}$and $h_{-}$. That is, each word represented by $l$ is cyclically reduced. But a word determined by $l$ already contains both $x^{2}$ and $z^{2}$, and so $l$ cannot represent a primitive element of $\pi_{1}(W)$ by Proposition 2.1 again.

\section{Primitive disks in a handlebody}

Recall that $(V, W ; \Sigma)$ denotes a genus-two Heegaard splitting of a lens space $L=L(p, q)$ with $p \geq 2$. We call an essential disk $E$ in $V$ primitive if there exists an essential disk $E^{\prime}$ in $W$ such that $\partial E$ intersects $\partial E^{\prime}$ transversely in a single point. Such a disk $E^{\prime}$ is called a dual disk of $E$. Note that $E^{\prime}$ is also primitive in $W$ with a dual disk $E$, and $W \cup \operatorname{Nbd}(E)$ and $V \cup \operatorname{Nbd}\left(E^{\prime}\right)$ are both solid tori. Primitive disks are necessarily nonseparating. We call a pair of disjoint, nonisotopic primitive disks in $V$ a primitive pair in $V$. Similarly, a triple of pairwise disjoint, nonisotopic, primitive disks (if it exists) is a primitive triple.

A nonseparating disk $E_{0}$ properly embedded in $V$ is called semiprimitive if there is a primitive disk $E^{\prime}$ in $W$ such that $\partial E^{\prime}$ is disjoint from $\partial E_{0}$. With a suitable choice of oriented meridian and longitude circles on the boundary of the solid torus obtained by cutting up $W$ along $E^{\prime}$, the oriented boundary circle $\partial E_{0}$ can be considered a $(p, 1)$-curve on the boundary of the solid torus, if $q=1$.

Any simple closed curve on the boundary of $W$ represents an element of $\pi_{1}(W)$, which is the free group of rank two. We can interpret primitive disks algebraically as follows, which is a direct consequence of [Gordon 1987]:

Lemma 3.1. Let $D$ be a nonseparating disk in $V$. Then $D$ is primitive if and only if $\partial D$ represents a primitive element of $\pi_{1}(W)$.

Note that no disk can be both primitive and semiprimitive since the boundary circle of a semiprimitive disk in $V$ represents the $p$-th power of a primitive element of $\pi_{1}(W)$. 
Let $D$ and $E$ be essential disks in $V$, and suppose that $D$ intersects $E$ transversely and minimally. Let $C \subset D$ be a disk cut up from $D$ by an outermost $\operatorname{arc} \beta$ of $D \cap E$ in $D$ such that $C \cap E=\beta$. We call such a $C$ an outermost subdisk of $D$ cut up by $D \cap E$. The $\operatorname{arc} \beta$ cuts $E$ into two disks, say $G$ and $H$. Then we have two essential disks $E_{1}$ and $E_{2}$ in $V$ which are isotopic to disks $G \cup C$ and $H \cup C$ respectively. We call $E_{1}$ and $E_{2}$ the disks from surgery on $E$ along the outermost subdisk $C$ of $D$ cut up by $D \cap E$. Observe that $E_{1}$ and $E_{2}$ each have fewer arcs of intersection with $D$ than $E$ had, since at least the arc $\beta$ no longer counts.

Since $E$ and $D$ are assumed to intersect minimally, $E_{1}$ and $E_{2}$ are isotopic to neither $E$ nor $D$. In particular, if both $D$ and $E$ are nonseparating, then the resulting disks $E_{1}$ and $E_{2}$ are both nonseparating and they are not isotopic to each other. Further, $E_{1}$ and $E_{2}$ are meridian disks of the solid torus $V$ cut up by $E$, and the boundary circles $\partial E_{1}$ and $\partial E_{2}$ are not isotopic to each other in the two holed torus $\partial V$ cut up by $\partial E$.

Theorem 3.2. Let $(V, W ; \Sigma)$ be the genus-two Heegaard splitting of the lens space $L=L(p, 1)$ with $p \geq 2$. Let $D$ and $E$ be primitive disks in $V$ which intersect each other transversely and minimally. Then one of the two disks from surgery on $E$ along an outermost subdisk of $D$ cut up by $D \cap E$ is primitive. Furthermore, it has a common dual disk with $E$.

Proof. We will prove the theorem only for $p \geq 5$. The cases of $p \in\{2,3,4\}$ will be similar but simpler.

Let $C$ be an outermost subdisk of $D$ cut up by $D \cap E$. The choice of a dual disk $E^{\prime}$ of $E$ determines a unique semiprimitive disk $E_{0}$ in $V$, namely, the meridian disk $E_{0}$ of $V$ disjoint from $E \cup E^{\prime}$. Among all the dual disks of $E$, choose one, denoted by $E^{\prime}$ again, so that the semiprimitive $E_{0}$ determined by $E^{\prime}$ intersects $C$ minimally. Further, there is a unique semiprimitive disk $E_{0}^{\prime}$ in $W$ disjoint from $E \cup E^{\prime}$. We give symbols $x$ and $y$ to oriented $\partial E^{\prime}$ and $\partial E_{0}^{\prime}$ respectively to have $\pi_{1}(W)=\langle x, y\rangle$. For convenience, we simply identify the boundary circles $\partial E^{\prime}$ and $\partial E_{0}^{\prime}$ with the assigned symbols $x$ and $y$ respectively. Notice that the circle $y$ is disjoint from $\partial E$ and intersects $\partial E_{0}$ in $p$ points in the same direction, and $x$ is disjoint from $\partial E_{0}$ and intersects $\partial E$ in a single point. Thus we may assume that $\partial E_{0}$ and $\partial E$ determine the words $y^{p}$ and $x$ respectively.

Let $\Sigma_{0}$ be the 4-holed sphere $\partial V$ cut up by $\partial E \cup \partial E_{0}$. We regard $\Sigma_{0}$ as a 2-holed annulus where the two boundary circles came from $\partial E_{0}$ and the two holes came from $\partial E$. Then $y \cap \Sigma_{0}$ is the union of $p$ spanning arcs which cut $\Sigma_{0}$ into $p$ rectangles, and $x$ is a single arc connecting two holes which are contained in a single rectangle. See Figure 2, left.

Suppose first that $C$ is disjoint from $E_{0}$. Note that one of the disks from surgery on $E$ along $C$ is $E_{0}$, which is semiprimitive. The $\operatorname{arc} C \cap \Sigma_{0}$ is the frontier of 

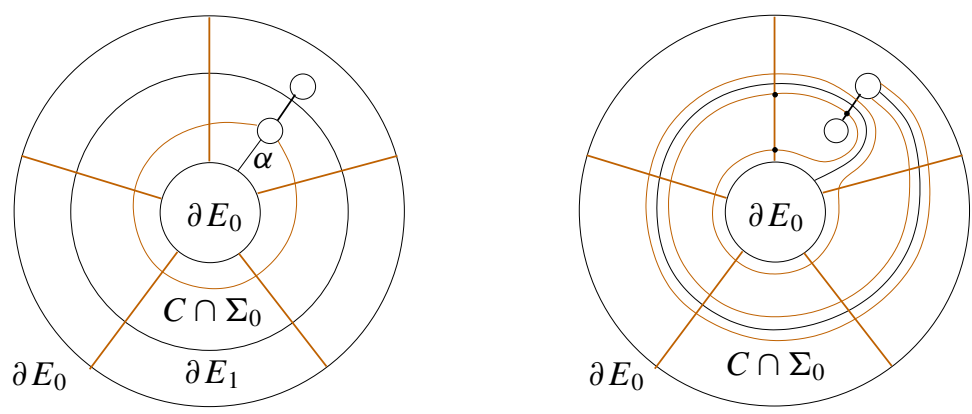

Figure 2. The 2-holed annulus $\Sigma_{0}$ in $L(5,1)$.

a regular neighborhood of the union of one boundary circle of $\Sigma_{0}$ and an arc $\alpha$ connecting the boundary circle to a hole. Observe that the $\operatorname{arc} \alpha$ is disjoint from $y \cap \Sigma_{0}$, otherwise a word of $\partial D$ must contain $y x y^{-1}$ (after changing orientation if necessary) which contradicts that $D$ is primitive, by Lemma 2.2. See Figure 2, right. Consequently, if we denote by $E_{1}$ the disk from surgery that is not $E_{0}$, then $\partial E_{1}$ intersects $\partial E^{\prime}$ in a single point. That is, the resulting disk $E_{1}$ is primitive with the common dual disk $E^{\prime}$ of $E$. See Figure 2, left.

From now on, we assume that $C$ intersects $E_{0}$. Let $C_{0}$ be an outermost subdisk of $C$ cut up by $C \cap E_{0}$. The arc $C_{0} \cap \Sigma_{0}$ is the frontier of a regular neighborhood of one hole of $\Sigma_{0}$ and an arc, say $\alpha_{0}$, connecting the hole to a boundary circle of $\Sigma_{0}$. By the same reasoning as in the case of $\alpha$, the arc $\alpha_{0}$ is disjoint from $y \cap \Sigma_{0}$. Thus one of the disks from surgery on $E_{0}$ along $C_{0}$ is $E$, and the other one, denoted by $E_{1}$ again, is primitive since $\partial E_{1}$ intersects $\partial E^{\prime}$ in a single point as in the previous case. Note that $\left|C \cap E_{1}\right|<\left|C \cap E_{0}\right|$ from the surgery construction. See $\Sigma_{0}$ in Figure 3.

Let $\Sigma_{1}$ be the 4-holed sphere $\partial V$ cut up by $\partial E \cup \partial E_{1}$. We regard $\Sigma_{1}$ as a 2-holed annulus, like $\Sigma_{0}$, where the two boundary circles came from $\partial E_{1}$ and the two holes came from $\partial E$. Then $y \cap \Sigma_{1}$ is the union of $p$ spanning arcs which cut $\Sigma_{1}$ into $p$ rectangles as in the case of $\Sigma_{0}$, but the two holes, which came from $\partial E$, are now contained in different consecutive rectangles, and $x \cap \Sigma_{1}$ is the union of two arcs each joining a hole and a boundary circle of $\Sigma_{1}$ as in Figure 3. If the original subdisk $C$ is disjoint from $E_{1}$, then we are done since $E_{1}$ is the desired primitive disk resulting from the surgery.

Suppose that $C$ also intersects $E_{1}$, and let $C_{1}$ be an outermost subdisk of $C$ cut up by $C \cap E_{1}$. Then $C_{1} \cap \Sigma_{1}$ is the frontier of a regular neighborhood of the union of one hole of $\Sigma_{1}$ and an arc, say $\alpha_{1}$, connecting the hole to a boundary circle. The arc $\alpha_{1}$ is also disjoint from $y \cap \Sigma_{1}$ by the same reasoning as for $\alpha_{0}$. Thus if we denote by $E_{2}$ the disk from surgery on $E_{1}$ along $C_{1}$ that is not $E$, then $\partial E_{2}$ represents a word $x y x y^{p-1}$. See $\Sigma_{1}$ in Figure 3. 

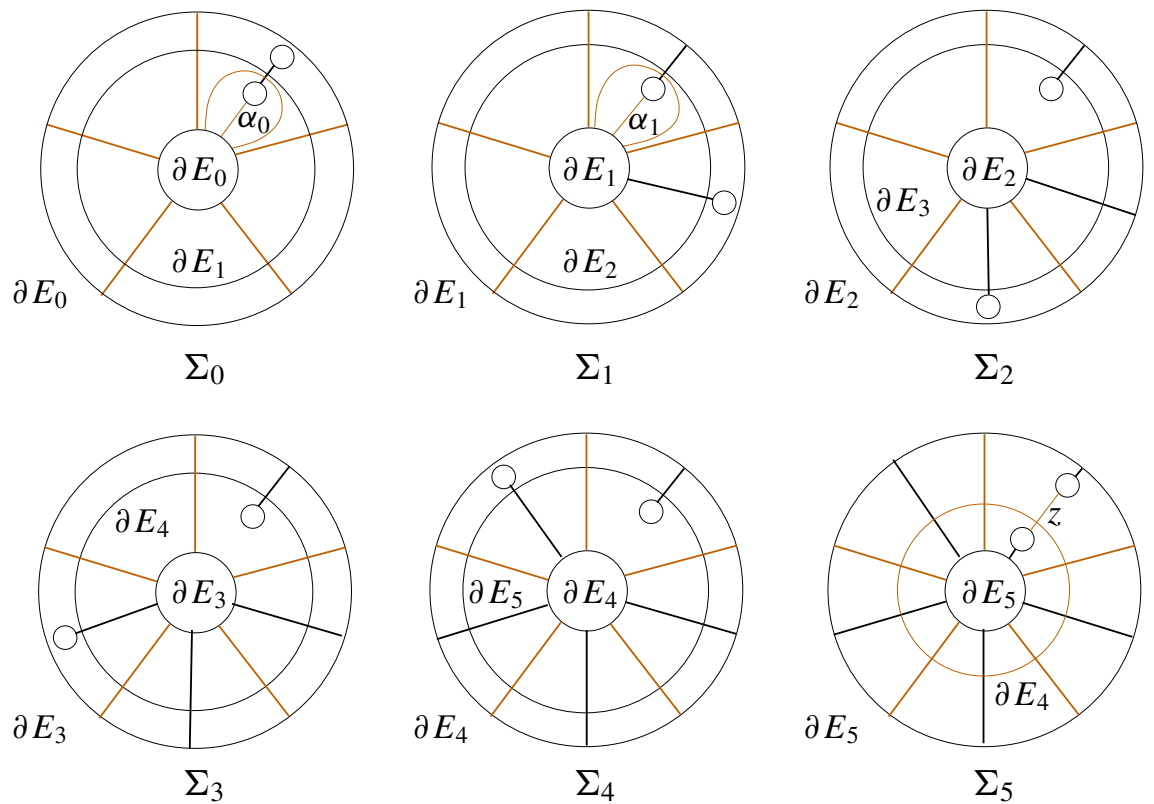

Figure 3. The sequence of 2-holed annuli from the consecutive surgeries for $L(5,1)$.

We continue such a construction repeatedly whenever $C$ also intersects the next disk. For each $1 \leq j \leq p-1$, if $C$ intersects $E_{j}$, then we obtain the disk $E_{j+1}$ from surgery on $E_{j}$ along an outermost subdisk $C_{j}$ cut up by $C \cap E_{j}$. We see that $\left|C \cap E_{j+1}\right|<\left|C \cap E_{j}\right|$ from the surgery construction. In the 2-holed annulus $\Sigma_{j}$, the arc $C_{j} \cap \Sigma_{j}$ is the frontier of a regular neighborhood of the union of a hole of $\Sigma_{j}$ and an $\operatorname{arc} \alpha_{j}$ connecting the hole to a boundary circle. The $\operatorname{arc} \alpha_{j}$ is disjoint from $y \cap \Sigma_{j}$, and so $\partial E_{j+1}$ represents a word of the form $(x y)^{j} x y^{p-j}$. In particular, notice that the disk $E_{p}$ is semiprimitive and $E_{p-1}$ is primitive, since there is a primitive disk $E^{\prime \prime}$ in $W$ disjoint from $\partial E_{p}$ that intersects $\partial E_{p-1}$ in a single point. Such an $E^{\prime \prime}$ is not hard to find. In the final 2-holed annulus $\Sigma_{5}$ in Figure 3, the arc $z$ is the boundary circle of $E^{\prime \prime}$ in $\Sigma_{p}$. Note that $z$ is disjoint from $x \cup y$, and so it does bound a disk $E^{\prime \prime}$ in the 3-ball $W$ cut up by $E^{\prime} \cup E_{0}^{\prime}$. Also, $z$ intersects $\partial E_{p-1}$ in a single point and is disjoint from $\partial E_{p}$.

We remark that each of the $\operatorname{arcs} \alpha_{j}, j \in\{0,1, \ldots, p-1\}$, is disjoint from the circle $y$ due to the fact that $D$ is primitive. There are infinitely many $\operatorname{arcs} \alpha_{0}$ that are not isotopic to each other in $\Sigma_{0}$, but each arc $\alpha_{j}$ in $\Sigma_{j}$ with $j \geq 1$ is unique up to isotopy. Therefore, once $E_{1}$ is determined, we have the unique sequence of disks $E_{2}, E_{3}, \ldots, E_{p}$ only under the condition that each $\alpha_{j}$ is disjoint from $y$.

Claim. For each $j \in\{2,3, \ldots, p-1\}$, the subdisk $C$ intersects $E_{j}$. 
Proof of claim. Suppose not, and let $E_{j}$ be the first disk disjoint from $C$. First, suppose that $j \in\{2,3, \ldots, p-3\}$. Then $C$ is disjoint from $E_{j}$ and intersects $E_{j-1}$, and so the arc $\partial C \cap \Sigma_{j}$ gives a subword of $\partial D$ of the form $(y x)^{j} y^{p-j}$ which implies that $D$ is not primitive by Lemma 2.2 again, which is a contradiction. Next, suppose that $j=p-2$. That is, $C$ is disjoint from $E_{p-2}$ and intersects $E_{p-3}$. Then one of the resulting disks from surgery on $E$ along $C$ is $E_{p-2}$, and the other one is exactly $E_{p-1}$, which is a disk in the sequence of disks in the previous construction. The subdisk $C$ is disjoint from $E_{p-2} \cup E_{p-1}$, and consequently, $C$ necessarily intersects the semiprimitive disk $E_{p}$ in the previous construction in a single arc. That is, $\left|C \cap E_{p}\right|=1$. But from the consecutive surgery constructions for $j \in\{2,3, \ldots, p-3\}$, we have $1 \leq\left|C \cap E_{p-3}\right|<\left|C \cap E_{0}\right|$, which contradicts the minimality of $\left|C \cap E_{0}\right|$. Similarly, if $j=p-1$, then we have the same contradiction on the minimality, since $C$ is disjoint from $E_{p}$ in this case. This proves the claim.

By the claim, we can do surgery on $E_{p-1}$ along $C_{p-1}$ and one resulting disk from surgery is $E_{p}$, the semiprimitive disk. But $\left|C \cap E_{j+1}\right|<\left|C \cap E_{j}\right|$ for each $j \in\{1,2, \ldots, p-1\}$, and consequently $\left|C \cap E_{p}\right|<\left|C \cap E_{0}\right|$, which contradicts the minimality of $\left|C \cap E_{0}\right|$ again.

Therefore the primitive disk $E_{1}$ is a disk from surgery on $E$ along $C$, and $E^{\prime}$ is also a dual disk of $E_{1}$, and so we complete the proof. We note that the other disk from surgery is either $E_{0}$ or $E_{2}$ depending on whether $C$ is disjoint from $E_{0}$ or not.

Theorem 3.3. Let $(V, W ; \Sigma)$ be the genus-two Heegaard splitting of the lens space $L=L(p, 1)$ with $p \geq 2$. Then, for every primitive pair $\{D, E\}$ of $V, D$ and $E$ have a common dual disk. In particular, the two disks of each primitive pair have a unique common dual disk if $p \geq 3$, and have exactly two common dual disks if $p=2$ which form a primitive pair in $W$.

Proof. The proof of the existence of a common dual disk goes almost in the same way as that of Theorem 3.2, by taking the primitive disk $D$ disjoint from $E$ instead of the outermost subdisk $C$ in Theorem 3.2. That is, when we choose a dual disk $E^{\prime}$ of $E$ so that $\left|\partial D \cap \partial E_{0}\right|$ is minimal where $E_{0}$ is the unique semiprimitive disk in $V$ disjoint from $\operatorname{Nbd}\left(E \cup E^{\prime}\right)$, the primitive disk $D$ must be $E_{1}$, having the common dual disk $E^{\prime}$ of $E$.

Now, let $E^{\prime}$ be a common dual disk of $D$ and $E$. Let $E_{0}$ and $E_{0}^{\prime}$ be the unique meridian disks of $V$ and $W$ respectively that are disjoint from $\operatorname{Nbd}\left(E \cup E^{\prime}\right)$ (see Figure 4, left). Cut the surface $\Sigma$ along $\partial E^{\prime} \cup \partial E_{0}^{\prime}$ to obtain the 4-holed sphere $\Sigma^{\prime}$. Then $\partial E \cap \Sigma^{\prime}$ is a single arc in $\Sigma^{\prime}$ connecting the two holes coming from $\partial E^{\prime}$, and $\partial D \cap \Sigma^{\prime}$ consists of $p-1$ parallel arcs connecting the two holes coming from $\partial E_{0}^{\prime}$ and two arcs connecting the holes coming from $\partial E^{\prime}$ to $\partial E_{0}^{\prime}$ on opposite sides, as in Figure 4. 

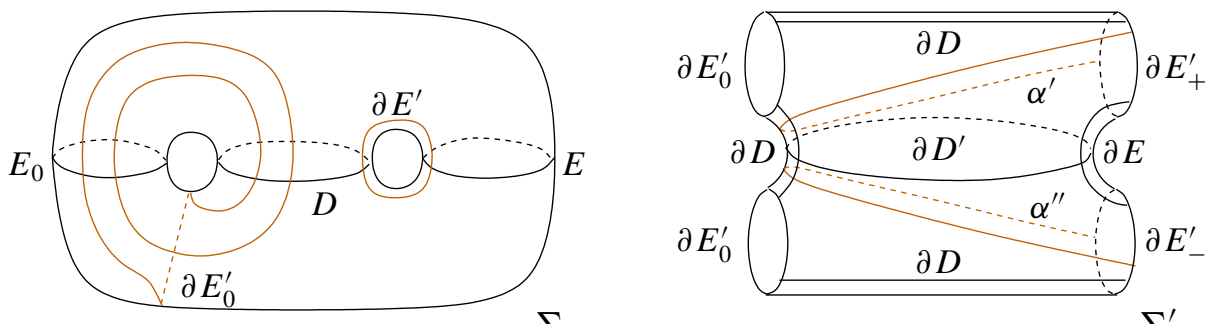

$\Sigma$

$\Sigma^{\prime}$

Figure 4. The surfaces $\Sigma$ and $\Sigma^{\prime}$ for $L(2,1)$.

Let $D^{\prime}$ be a common dual disk of $D$ and $E$ which is not isotopic to $E^{\prime}$. Then an outermost subdisk $C^{\prime}$ of $D^{\prime}$ cut up by $D^{\prime} \cap\left(E^{\prime} \cup E_{0}^{\prime}\right)$ would intersect $\partial D$ if $C^{\prime}$ is incident to $E^{\prime}$. Denote by $\partial E_{+}^{\prime}$ and $\partial E_{-}^{\prime}$ the two holes of $\Sigma^{\prime}$ which came from $\partial E^{\prime}$. We may assume that the endpoints of the $\operatorname{arc} \alpha^{\prime}=C^{\prime} \cap \Sigma^{\prime}$ meet $\partial E_{+}^{\prime}$. Since $\left|\partial D^{\prime} \cap \partial E_{+}^{\prime}\right|=\left|\partial D^{\prime} \cap \partial E_{-}^{\prime}\right|$, we must have one more arc component $\alpha^{\prime \prime}$ of $\partial D^{\prime} \cap \Sigma^{\prime}$ other than $C^{\prime} \cap \Sigma^{\prime}$ whose endpoints meet $\partial E_{-}^{\prime}$ (see Figure 4, right). The arc $\alpha^{\prime \prime}$ also intersects $\partial D$, and so $\partial D^{\prime}$ intersects $\partial D$ in more than one point, which contradicts that $D^{\prime}$ is a dual disk of $D$. Similarly, if $C^{\prime}$ is incident to $E_{0}^{\prime}$, then $D^{\prime}$ cannot be a dual disk of $E$. Thus we see that $D^{\prime}$ is disjoint from $E^{\prime} \cup E_{0}^{\prime}$.

If $p \geq 3$, there is no possibility of such a disk $D^{\prime}$ which is disjoint from $E^{\prime} \cup E_{0}^{\prime}$ and is not isotopic to $E^{\prime}$, and so $E^{\prime}$ is the unique common dual disk. If $p=2$, there is a unique circle in $\Sigma^{\prime}$ which is not boundary parallel and which intersects $\partial E$ and $\partial D$ exactly once (see the circle $\partial D^{\prime}$ in Figure 4 , right). So we have exactly two common dual disks $D^{\prime}$ and $E^{\prime}$ and in this case they are disjoint from each other.

Given a primitive disk $D$ in $V$, there are infinitely many (nonisotopic) primitive disks each of which forms a primitive pair together with $D$. But any primitive pair can be contained in at most one primitive triple, proved as follows:

Theorem 3.4. Let $(V, W ; \Sigma)$ be the genus-two Heegaard splitting of the lens space $L=L(p, 1)$ with $p \geq 2$. Then there is a primitive triple of $V$ if and only if $p=3$. In this case, every primitive pair is contained in a unique primitive triple.

Proof. Let $\left\{E, E_{1}\right\}$ be a primitive pair of $V$. Choose a common dual disk $E^{\prime}$ of $E$ and $E_{1}$ given by Theorem 3.3. There are unique semiprimitive disks $E_{0}$ in $V$ and $E_{0}^{\prime}$ in $W$ disjoint from $\operatorname{Nbd}\left(E \cup E^{\prime}\right)$. Let $\Sigma_{1}$ be the 4-holed sphere $\partial V$ cut up by $\partial E \cup \partial E_{1}$, and as in Figure 3 again, consider $\Sigma_{1}$ as a 2-holed annulus with two boundary circles coming from $\partial E_{1}$ and two holes from $\partial E$. We give symbols $x$ and $y$ to $\partial E^{\prime}$ and $\partial E_{0}^{\prime}$ respectively as in the proof of Theorem 3.2.

The boundary of any primitive disk $E_{2}$ in $V$ disjoint from $E$ and $E_{1}$, if it exists, lies in $\Sigma_{1}$, and it is the frontier of a regular neighborhood of the union of a boundary circle, a hole of $\Sigma_{1}$ and an arc $\alpha_{1}$ connecting them. This arc is disjoint from the 


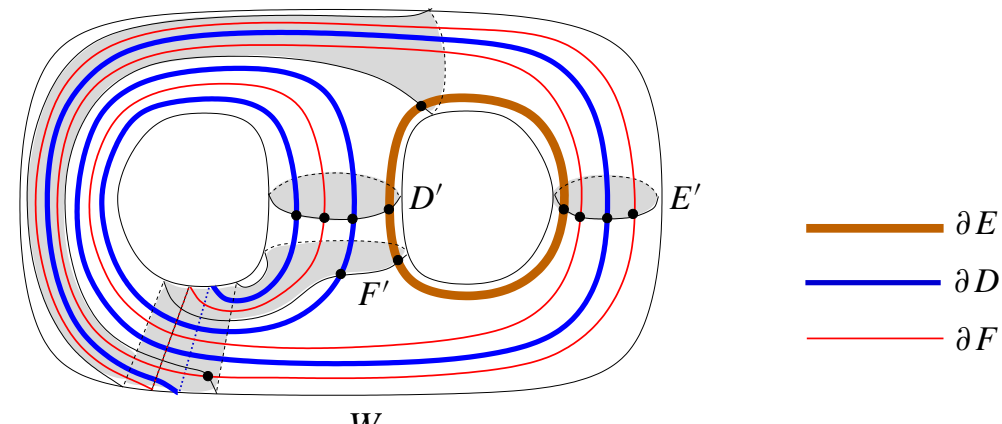

Figure 5. The primitive triple $\left\{D^{\prime}, E^{\prime}, F^{\prime}\right\}$ of $W$ in $L(3,1)$ with the boundary circles $\partial D, \partial E$, and $\partial F$ of the disks in the primitive triple of $V$.

arcs $y \cap \Sigma_{1}$, otherwise $\partial E_{2}$ represents a word containing $y x y^{-1}$; that is, $E_{2}$ is not primitive. Consequently, $\partial E_{2}$ is uniquely determined and it represents a word of the form $x y x y^{p-1}$, and so it is primitive if and only if $p=3$. Thus, only when $p=3$, we have the unique primitive triple $\left\{E, E_{1}, E_{2}\right\}$ containing the pair $\left\{E, E_{1}\right\}$.

Remark 3.5. For any primitive triple $\{D, E, F\}$ of $V$ in $L(3,1)$, by Theorem 3.3, there exist unique common dual disks $D^{\prime}, E^{\prime}$, and $F^{\prime}$ of the disks in the pairs $\{E, F\}$, $\{F, D\}$, and $\{D, E\}$ respectively. In fact, the disks $D^{\prime}, E^{\prime}$, and $F^{\prime}$ form a primitive triple of $W$. Furthermore, we have $\left|\partial D^{\prime} \cap \partial D\right|=\left|\partial E^{\prime} \cap \partial E\right|=\left|\partial F^{\prime} \cap \partial F\right|=2$. Figure 5 illustrates the triple $\left\{D^{\prime}, E^{\prime}, F,\right\}$ of $W$ together with the boundary circles of $D, E$ and $F$ in $\partial W=\Sigma$.

\section{The complex of primitive disks}

Let $M$ be an irreducible 3-manifold with compressible boundary. The disk complex of $M$ is a simplicial complex defined as follows: The vertices of the disk complex are isotopy classes of essential disks in $M$, and a collection of $k+1$ vertices spans a $k$-simplex if and only if it admits a collection of representative disks which are pairwise disjoint. In particular, if $M$ is a handlebody of genus $g \geq 2$, then the disk complex is $(3 g-4)$-dimensional and is not locally finite. The following is a key property of a disk complex:

Theorem 4.1. If Y is a full subcomplex of the disk complex satisfying the following condition, then T⿱ is contractible:

Let $E$ and $D$ be disks in $M$ representing vertices of $\mathscr{K}$. If $E$ and $D$ intersect transversely and minimally, then at least one of the disks from surgery on $E$ along an outermost subdisk of $D$ cut up by $D \cap E$ represents a vertex of $\mathcal{K}$. 

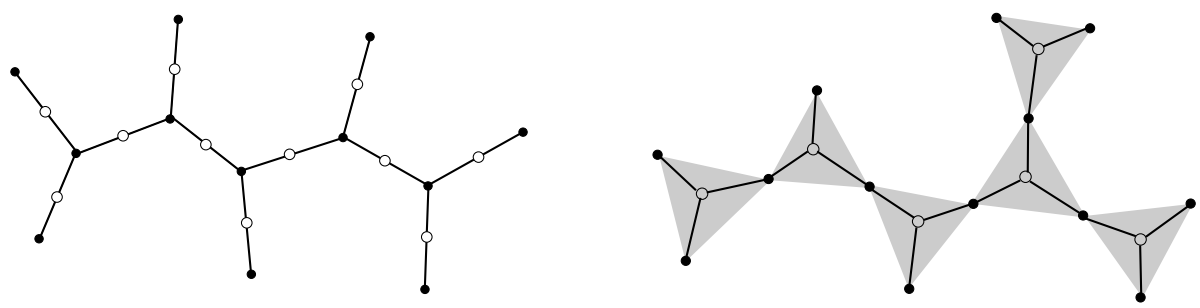

Figure 6. Small portions of primitive disk complexes $\mathscr{P}(V)$ for $p \neq 3$ (left) and $p=3$ (right).

In [Cho 2008], the above theorem is proved in the case that $M$ is a handlebody, but the proof is still valid for an arbitrary irreducible manifold with compressible boundary. From the theorem, we see that the disk complex itself is contractible.

Now consider the genus-two Heegaard splitting $(V, W ; \Sigma)$ of a lens space $L(p, 1)$ with $p \geq 2$. We define the primitive disk complex, denoted by $\mathscr{P}(V)$, to be the full subcomplex of the disk complex spanned by the vertices of primitive disks in $V$. We already know that every primitive disk is a member of infinitely many primitive pairs, and so every vertex of $\mathscr{P}(V)$ has infinite valency. The following is our main theorem, a direct consequence of Theorems 3.2, 3.4 and 4.1:

Theorem 4.2. Let $(V, W ; \Sigma)$ be the genus-two Heegaard splitting of the lens space $L=L(p, 1)$ with $p \geq 2$. The primitive disk complex $\mathscr{P}(V)$ is contractible. In particular, if $p \neq 3$ it is a tree, and if $p=3$ it is 2-dimensional and every edge is contained in a unique 2-simplex.

Figure 6 illustrates portions of the primitive disk complexes. The black vertices are the vertices of $\mathscr{P}(V)$ while the white ones are the barycenters of edges when $p \neq 3$ and of 2-simplices when $p=3$. Observe that the 2-dimensional $\mathscr{P}(V)$ deformation retracts to a tree in its barycentric subdivision, as in the figure.

\section{Genus-two Goeritz groups of lens spaces $L(p, 1)$}

In this section, we give explicit presentation of the genus-two Goeritz group $\mathscr{G}$ of each lens space $L(p, 1)$. From Theorem 4.2, if $p \neq 3$, the primitive disk complex $\mathscr{P}(V)$ is a tree, and if $p=3$, then $\mathscr{P}(V)$ is 2-dimensional but deformation retracts to a tree. We simply denote by $\mathscr{T}$ the barycentric subdivision of the tree $\mathscr{P}(V)$ if $p \neq 3$ and the deformation retract of $\mathscr{P}(V)$ if $p=3$. Each of the trees $\mathscr{T}$ is bipartite, as in Figure 6, with the black vertices of (countably) infinite valence, and the white vertices of valence 2 if $p \neq 3$ and of valence 3 if $p=3$.

Each black vertex of $\mathscr{T}$ is represented by a primitive disk, while each white vertex is represented by a primitive pair if $p \neq 3$ and by a primitive triple if $p=3$. 

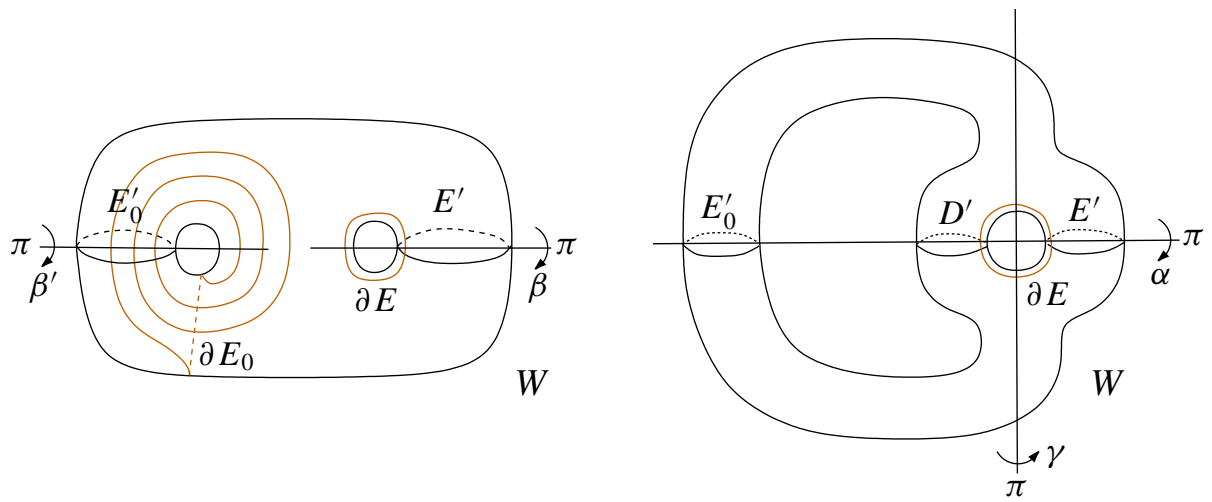

Figure 7. Generators of the stabilizer subgroup $\mathscr{G}_{\{E\}}$.

An element of the group $\mathscr{G}$ can be considered a simplicial automorphism of $\mathscr{T}$. The tree $\mathscr{T}$ is invariant under the action of $\mathscr{G}$ for each $L(p, 1)$. In particular, $\mathscr{G}$ acts transitively on the set of black vertices and on the set of white vertices, and hence the quotient of $\mathscr{T}$ by the action of $\mathscr{G}$ is a single edge of which one end vertex is black and another one white. Thus, by the theory of groups acting on trees due to Bass and Serre (see [Serre 1980]), the group $\varphi$ can be expressed as the free product of the stabilizer subgroups of two end vertices with the amalgamated stabilizer subgroup of the edge.

First, we find a presentation of the stabilizer subgroup of a black vertex of $\mathscr{T}$; that is, of (the isotopy class of) a primitive disk in $V$. For convenience, we will not distinguish disks (pairs and triples of disks) and homeomorphisms from their isotopy classes in their notations. Throughout the section, $\varphi_{\left\{A_{1}, A_{2}, \ldots, A_{k}\right\}}$ will denote the subgroup of $\mathscr{G}$ of elements preserving $A_{1}, A_{2}, \ldots, A_{k}$ setwise, where $A_{i}$ will be (isotopy classes of) disks or unions of disks in $V$ or in $W$.

Lemma 5.1. Let $E$ be a primitive disk in $V$. The stabilizer subgroup $\mathscr{G}_{\{E\}}$ of $E$ has the presentation $\left\langle\alpha \mid \alpha^{2}=1\right\rangle \oplus\left\langle\beta, \gamma \mid \gamma^{2}=1\right\rangle$, where the generators $\alpha, \beta$ and $\gamma$ are described in Figure 7.

Proof. Let $\mathscr{P}^{\prime}(W)$ be the full subcomplex of the primitive disk complex $\mathscr{P}(W)$ for $W$ spanned by the vertices of dual disks of $E$. There is a unique semiprimitive disk $E_{0}^{\prime}$ in $W$ disjoint from $\partial E$, and it is easy to show that any dual disk of $E$ is disjoint from $E_{0}^{\prime}$. Thus $\mathscr{P}^{\prime}(W)$ is 1-dimensional and further, by a similar argument used for $\mathscr{P}(V)$, we have that $\mathscr{P}^{\prime}(W)$ is a tree whose vertices have infinite valence. That is, when two dual disks of $E$ intersect each other, one of the two disks from the surgery construction is $E_{0}^{\prime}$ and the other one is again a dual disk of $E$. Denote by $\mathscr{T}^{\prime}$ the barycentric subdivision of $\mathscr{P}^{\prime}(W)$. The tree $\mathscr{T}^{\prime}$ is invariant under the action of the stabilizer subgroup $\mathscr{G}_{\{E\}}$, and the quotient of $\mathscr{T}^{\prime}$ by the action is a single edge. One vertex of this edge corresponds to a dual disk $E^{\prime}$ of $E$, and the other one to a 
primitive pair $\left\{E^{\prime}, D^{\prime}\right\}$ of dual disks of $E$. Thus $\mathscr{G}_{\{E\}}$ can be expressed as the free product of the stabilizer subgroups $\mathscr{G}_{\left\{E, E^{\prime}\right\}} * \mathscr{G}_{\left\{E, E^{\prime} \cup D^{\prime}\right\}}$ amalgamated by $\mathscr{G}_{\left\{E, E^{\prime}, D^{\prime}\right\}}$.

Consider the subgroup $\mathscr{G}_{\left\{E, E^{\prime}\right\}}$ first. Any element of $\mathscr{G}_{\left\{E, E^{\prime}\right\}}$ also preserves the disks $E_{0}$ and $E_{0}^{\prime}$, which are unique meridian disks disjoint from $E \cup E^{\prime}$ in $V$ and in $W$ respectively. Since $V$ cut up by $E \cup E_{0}$ and $W$ cut up by $E^{\prime} \cup E_{0}^{\prime}$ are all 3-balls, the group $\varphi_{\left\{E, E^{\prime}\right\}}$ is identified with the group of isotopy classes of orientationpreserving homeomorphisms of $\Sigma=\partial V=\partial W$ which preserve $\partial E, \partial E^{\prime}, \partial E_{0}$, and $\partial E_{0}^{\prime}$. This group has a presentation $\left\langle\beta, \beta^{\prime} \mid\left(\beta \beta^{\prime}\right)^{2}=1, \beta \beta^{\prime}=\beta^{\prime} \beta\right\rangle$, where the generators $\beta$ and $\beta^{\prime}$ are $\pi$-rotations (half Dehn twists) described in Figure 7, left.

Next, consider the subgroup $\mathscr{G}_{\left\{E, E^{\prime} \cup D^{\prime}\right\}}$. Any element of this group preserves $E^{\prime} \cup D^{\prime}$ in $W$, and further it preserves $E$ and $E_{0} \cup D_{0}$ in $V$ where $E_{0}$ and $D_{0}$ are unique meridian disks in $V$ disjoint from $E \cup E^{\prime}$ and $E \cup D^{\prime}$ respectively. Thus $\mathscr{G}_{\left\{E, E^{\prime} \cup D^{\prime}\right\}}$ is generated by two elements $\alpha$ and $\gamma$, where $\alpha$ is the hyperelliptic involution, and $\gamma$ is the element of order 2 exchanging $E^{\prime}$ and $D^{\prime}$ described in Figure 7, right. Thus $\mathscr{G}_{\left\{E, E^{\prime} \cup D^{\prime}\right\}}$ has the presentation $\left\langle\alpha \mid \alpha^{2}=1\right\rangle \oplus\left\langle\gamma \mid \gamma^{2}=1\right\rangle$. Similarly, $\mathscr{G}_{\left\{E, E^{\prime}, D^{\prime}\right\}}$ has the presentation $\left\langle\alpha \mid \alpha^{2}=1\right\rangle$. Observing that $\alpha$ satisfies $\beta \beta^{\prime}=\alpha$, we have the desired presentation of $\mathscr{G}_{\{E\}}$.

Thus the stabilizer subgroups of black vertices have the same presentation for each $p \geq 2$, but for white vertices, we have the following cases depending on $p$ :

Lemma 5.2. A white vertex of $\mathcal{T}$ corresponds to a primitive pair if $p \neq 3$ and to a primitive triple if $p=3$.

(1) Let $\{D, E\}$ be a primitive pair of $V$ in $L(p, 1)$. Then the stabilizer subgroup $\mathscr{G}_{\{D \cup E\}}$ has the presentation $\left\langle\rho, \gamma \mid \rho^{4}=\gamma^{2}=(\rho \gamma)^{2}=1\right\rangle$ if $p=2$, and $\left\langle\alpha \mid \alpha^{2}=1\right\rangle \oplus\left\langle\sigma \mid \sigma^{2}=1\right\rangle$ if $p \geq 3$, where the generators are described in Figures 8 and 9 .
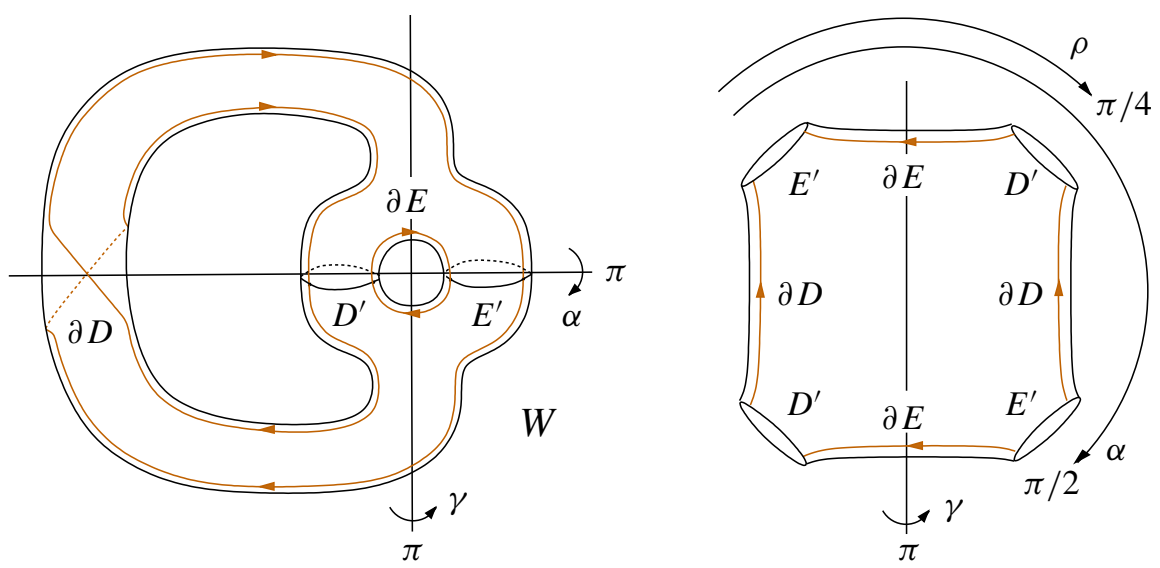

Figure 8. Generators of the stabilizer subgroup $\mathscr{G}_{\{D \cup E\}}$ for $L(2,1)$. 


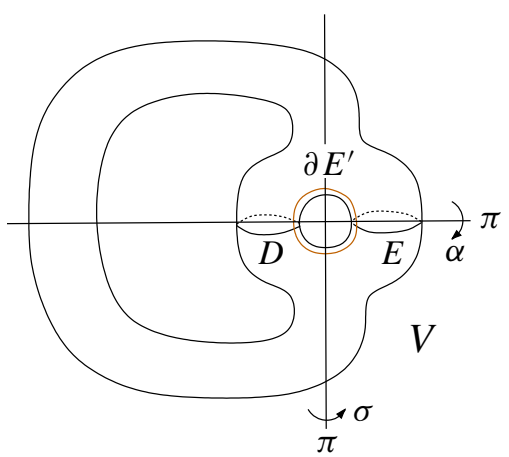

Figure 9. Generators of the stabilizer subgroup $\mathscr{G}_{\{D \cup E\}}$ for $L(p, 1)$, with $p \geq 3$.

(2) Let $\{D, E, F\}$ be a primitive triple of $V$ in $L(3,1)$. The stabilizer subgroup $\mathscr{G}_{\{D \cup E \cup F\}}$ has the presentation $\left\langle\alpha \mid \alpha^{2}=1\right\rangle \oplus\left\langle\delta, \gamma \mid \delta^{3}=\gamma^{2}=(\gamma \delta)^{2}=1\right\rangle$, where the generators are described in Figure 10.

Proof. (1) First, let $\{D, E\}$ be a primitive pair of $V$ in $L(2,1)$. Then, by Theorem 3.3, there is a unique primitive pair $\left\{D^{\prime}, E^{\prime}\right\}$ of $W$ such that $D^{\prime}$ and $E^{\prime}$ are common dual disks of $D$ and $E$. Any element of $\varphi_{\{D \cup E\}}$ preserves $D^{\prime} \cup E^{\prime}$, and hence $\varphi_{\{D \cup E\}}$ is identified with the stabilizer subgroup $\mathscr{G}_{\left\{D \cup E, D^{\prime} \cup E^{\prime}\right\}}$. Since $D \cup E$ and $D^{\prime} \cup E^{\prime}$ cut up $V$ and $W$ into 3-balls, the group $\mathscr{G}_{\left\{D \cup E, D^{\prime} \cup E^{\prime}\right\}}$ is identified with the group of isotopy classes of orientation-preserving homeomorphisms of $\Sigma=\partial V=\partial W$ which preserve $\partial D \cup \partial E$ and $\partial D^{\prime} \cup \partial E^{\prime}$. This is the dihedral group $D_{8}$ of order 8 with generators $\rho$ and $\gamma$ described in Figure 8. The 3-ball in Figure 8, right, is obtained by cutting up $W$ along $D^{\prime} \cup E^{\prime}$. Figure 8 gives two descriptions of the
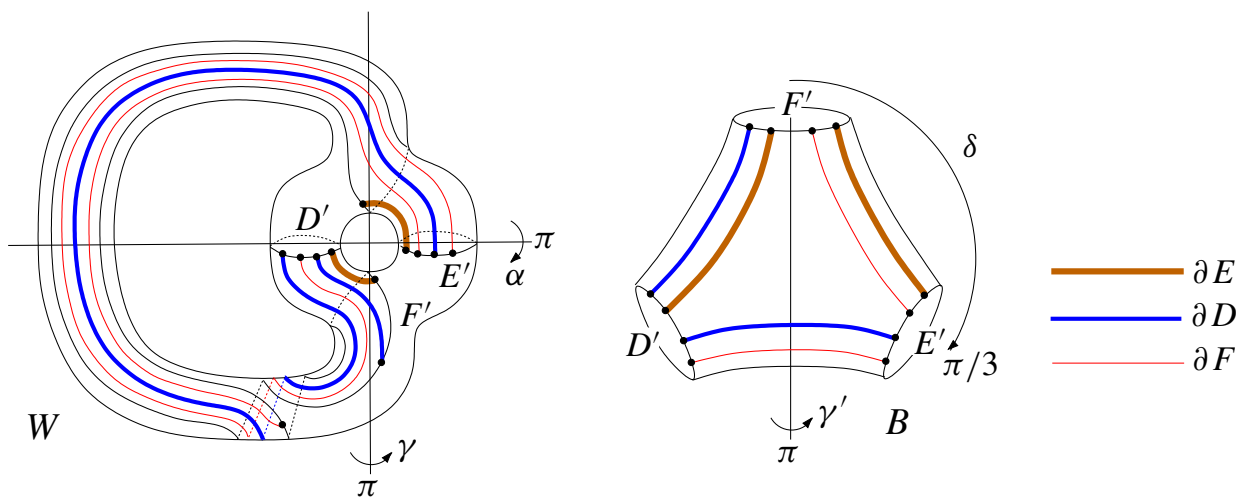

Figure 10. Left: The primitive triple $\left\{D^{\prime}, E^{\prime}, F^{\prime}\right\}$ of $W$ and the $\operatorname{arcs}(\partial D \cup \partial E \cup \partial F) \cap \partial B$. Right: The 3-ball $B$. 
elements $\alpha$ and $\gamma$. Thus we have the presentation $\left\langle\rho, \gamma \mid \rho^{4}=\gamma^{2}=(\rho \gamma)^{2}=1\right\rangle$. We remark that the hyperelliptic involution $\alpha$ equals $\rho^{2}$.

Next, let $\{D, E\}$ be a primitive pair of $V$ in $L(p, 1)$ with $p \geq 3$. There is a unique common dual disk $E^{\prime}$ of $D$ and $E$ by Theorem 3.3, and hence $\varphi_{\{D \cup E\}}$ is identified with the stabilizer subgroup $\mathscr{G}_{\left\{D \cup E, E^{\prime}\right\}}$. As in the case of $\mathscr{G}_{\left\{E, E^{\prime} \cup D^{\prime}\right\}}$ in the proof of Lemma 5.1, this group is generated by two elements: One is the hyperelliptic involution $\alpha$, and the other one is the element, denoted by $\sigma$, of order 2 exchanging $D$ and $E$ described in Figure 9. Thus we have the presentation $\left\langle\alpha \mid \alpha^{2}=1\right\rangle \oplus\left\langle\sigma \mid \sigma^{2}=1\right\rangle$.

(2) Let $\{D, E, F\}$ be a primitive triple of $V$ in $L(3,1)$. Then there exists a unique primitive triple $\left\{D^{\prime}, E^{\prime}, F^{\prime}\right\}$ of $W$ as described in Remark 3.5 and Figure 5. Thus the stabilizer subgroup $\mathscr{G}_{\{D \cup E \cup F\}}$ is identified with $\mathscr{G}_{\left\{D \cup E \cup F, D^{\prime} \cup E^{\prime} \cup F^{\prime}\right\}}$. The union of three disks $D^{\prime} \cup E^{\prime} \cup F^{\prime}$ cuts up $W$ into two 3-balls. One of them, say $B$, is shown in Figure 10, right. Consider the group of isotopy classes of orientation-preserving homeomorphisms of $B$ which preserve $D^{\prime} \cup E^{\prime} \cup F^{\prime}$ and $(\partial D \cup \partial E \cup \partial F) \cap \partial B$ on the boundary. This group is the dihedral group $D_{6}=\left\langle\delta, \gamma^{\prime} \mid \delta^{3}=\gamma^{\prime 2}=\left(\gamma^{\prime} \delta\right)^{2}=1\right\rangle$ of order 6 with generators $\delta$ and $\gamma^{\prime}$ in Figure 10, right. The element $\gamma$ in Figure 10, left, is different from $\gamma^{\prime}$, since $\gamma$ exchanges the two 3-balls. But they are related by $\gamma=\alpha \gamma^{\prime}$, where $\alpha$ is the hyperelliptic involution exchanging the two 3-balls as described in Figure 10, left. Thus we see that the relation $\left(\gamma^{\prime} \delta\right)^{2}=1$ in $D_{6}$ is equivalent to $(\gamma \delta)^{2}=1$. Since the elements $\alpha, \gamma$ and $\delta$ extend to elements of $\mathscr{G}_{\left\{D \cup E \cup F, D^{\prime} \cup E^{\prime} \cup F^{\prime}\right\}}$, this group can be considered as the extension of $D_{6}$ by $\left\langle\alpha \mid \alpha^{2}=1\right\rangle$ with relations $\alpha \gamma \alpha=\gamma$ and $\alpha \delta \alpha=\delta$. Thus we have the desired presentation of $\mathscr{G}_{\{D \cup E \cup F\}}$.

Finally, the stabilizer subgroups of an edge are calculated in a similar way.

Lemma 5.3. An edge of $\mathcal{T}$ corresponds to the pair of end vertices.

(i) Let $\{D, E\}$ be a primitive pair of $V$ in $L(p, 1)$. Then $\mathscr{G}_{\{E, D \cup E\}}=\mathscr{G}_{\{E, D\}}$ has a presentation $\left\langle\alpha \mid \alpha^{2}=1\right\rangle \oplus\left\langle\gamma \mid \gamma^{2}=1\right\rangle$ if $p=2$, and a presentation $\left\langle\alpha \mid \alpha^{2}=1\right\rangle$ if $p \geq 3$.

(ii) Let $\{D, E, F\}$ be a primitive triple of $V$ in $L(3,1)$. Then $\mathscr{G}_{\{E, D \cup E \cup F\}}=$ $\mathscr{G}_{\{E, D \cup F\}}$ has a presentation $\left\langle\alpha \mid \alpha^{2}=1\right\rangle \oplus\left\langle\gamma \mid \gamma^{2}=1\right\rangle$.

Combining Lemmas 5.1, 5.2, and 5.3, we obtain the main result.

Theorem 5.4. The genus- 2 Goeritz group $G$ of a lens space $L(p, 1)$ with $p \geq 2$ has the following presentations:

(i) $\left\langle\beta, \rho, \gamma \mid \rho^{4}=\gamma^{2}=(\gamma \rho)^{2}=\rho^{2} \beta \rho^{2} \beta^{-1}=1\right\rangle$ if $p=2$.

(ii) $\left\langle\alpha \mid \alpha^{2}=1\right\rangle \oplus\left\langle\beta, \delta, \gamma \mid \delta^{3}=\gamma^{2}=(\gamma \delta)^{2}=1\right\rangle$ if $p=3$.

(iii) $\left\langle\alpha \mid \alpha^{2}=1\right\rangle \oplus\left\langle\beta, \gamma, \sigma \mid \gamma^{2}=\sigma^{2}=1\right\rangle$ if $p \geq 4$. 


\section{Acknowledgments}

The author wishes to express his gratitude to Darryl McCullough and Yuya Koda for their helpful discussions, valuable advice, and comments. The main part of this work was carried out while the author was visiting the Korea Institute for Advanced Study (KIAS) in summer 2010. The author is grateful to the institute and to Professor Jaigyoung Choe for their warm hospitality and support. The author is also grateful to the referee for providing helpful comments that improved the presentation.

\section{References}

[Akbas 2008] E. Akbas, "A presentation for the automorphisms of the 3-sphere that preserve a genus two Heegaard splitting”, Pacific J. Math. 236:2 (2008), 201-222. MR 2009d:57029 Zbl 1157.57002

[Bonahon 1983] F. Bonahon, "Difféotopies des espaces lenticulaires", Topology 22:3 (1983), 305-314. MR 85d:57008 Zbl 0526.57009

[Bonahon and Otal 1983] F. Bonahon and J.-P. Otal, "Scindements de Heegaard des espaces lenticulaires”, Ann. Sci. École Norm. Sup. (4) 16:3 (1983), 451-466. MR 85c:57010 Zbl 0545.57002

[Cho 2008] S. Cho, "Homeomorphisms of the 3-sphere that preserve a Heegaard splitting of genus two", Proc. Amer. Math. Soc. 136:3 (2008), 1113-1123. MR 2009c:57029 Zbl 1149.57025

[Cho and Koda 2012] S. Cho and Y. Koda, "Primitive disk complexes for lens spaces", preprint, 2012. arXiv 1206.6243

[Goeritz 1933] L. Goeritz, "Die Abbildungen der Brezelfläche und der Volbrezel vom Geschlecht 2", Abh. Math. Sem. Univ. Hamburg 9:1 (1933), 244-259. Zbl 0007.08102

[Gordon 1987] C. M. Gordon, "On primitive sets of loops in the boundary of a handlebody", Topology Appl. 27:3 (1987), 285-299. MR 88k:57013 Zbl 0634.57007

[Koda 2011] Y. Koda, "Automorphisms of the 3-sphere that preserve spatial graphs and handlebodyknots”, preprint, 2011. arXiv 1106.4777

[Osborne and Zieschang 1981] R. P. Osborne and H. Zieschang, "Primitives in the free group on two generators", Invent. Math. 63:1 (1981), 17-24. MR 82i:20042 Zbl 0438.20017

[Rolfsen 1976] D. Rolfsen, Knots and links, Mathematics Lecture Series 7, Publish or Perish, Berkeley, CA, 1976. MR 58 \#24236 Zbl 0339.55004

[Scharlemann 2004] M. Scharlemann, "Automorphisms of the 3-sphere that preserve a genus two Heegaard splitting”, Bol. Soc. Mat. Mexicana (3) 10:Special Issue (2004), 503-514. MR 2007c:57020 Zbl 1095.57017

[Serre 1980] J.-P. Serre, Trees, Springer, Berlin, 1980. MR 82c:20083 Zbl 0548.20018

[Waldhausen 1968] F. Waldhausen, "Heegaard-Zerlegungen der 3-Sphäre", Topology 7 (1968), 195-203. MR 37 \#3576 Zbl 0157.54501

Received June 29, 2012. Revised January 9, 2013.

\section{SANGBUM CHO}

DEPARTMENT OF MATHEMATICS EDUCATION

HANYANG UNIVERSITY

SEOUL 133-791

SOUTH KOREA

scho@hanyang.ac.kr 


\title{
PACIFIC JOURNAL OF MATHEMATICS
}

\author{
msp.org/pjm
}

Founded in 1951 by E. F. Beckenbach (1906-1982) and F. Wolf (1904-1989)

\section{EDITORS}

V. S. Varadarajan (Managing Editor)

Department of Mathematics

University of California

Los Angeles, CA 90095-1555

pacific@math.ucla.edu

Paul Balmer

Department of Mathematics

University of California

Los Angeles, CA 90095-1555

balmer@math.ucla.edu

Daryl Cooper

Department of Mathematics

University of California

Santa Barbara, CA 93106-3080 cooper@math.ucsb.edu

Jiang-Hua $\mathrm{Lu}$

Department of Mathematics

Pokfulam Rd., Hong Kong jhlu@maths.hku.hk
The University of Hong Kong

Don Blasius

Department of Mathematics University of California

Los Angeles, CA 90095-1555

blasius@math.ucla.edu

Robert Finn

Department of Mathematics Stanford University

Stanford, CA 94305-2125

finn@math.stanford.edu

Sorin Popa

Department of Mathematics

University of California

Los Angeles, CA 90095-1555 popa@math.ucla.edu

Paul Yang

Department of Mathematics Princeton University

Princeton NJ 08544-1000

yang@math.princeton.edu

\section{PRODUCTION}

Silvio Levy, Scientific Editor, production@msp.org

\section{SUPPORTING INSTITUTIONS}

ACADEMIA SINICA, TAIPEI

CALIFORNIA INST. OF TECHNOLOGY

INST. DE MATEMÁTICA PURA E APLICADA

KEIO UNIVERSITY

MATH. SCIENCES RESEARCH INSTITUTE

NEW MEXICO STATE UNIV.

OREGON STATE UNIV.

\author{
STANFORD UNIVERSITY \\ UNIV. OF BRITISH COLUMBIA \\ UNIV. OF CALIFORNIA, BERKELEY \\ UNIV. OF CALIFORNIA, DAVIS \\ UNIV. OF CALIFORNIA, LOS ANGELES \\ UNIV. OF CALIFORNIA, RIVERSIDE \\ UNIV. OF CALIFORNIA, SAN DIEGO \\ UNIV. OF CALIF., SANTA BARBARA
}

\author{
Vyjayanthi Chari \\ Department of Mathematics \\ University of California \\ Riverside, CA 92521-0135 \\ chari@math.ucr.edu \\ Kefeng Liu \\ Department of Mathematics \\ University of California \\ Los Angeles, CA 90095-1555 \\ liu@math.ucla.edu \\ Jie Qing \\ Department of Mathematics \\ University of California \\ Santa Cruz, CA 95064 \\ qing@cats.ucsc.edu
}

These supporting institutions contribute to the cost of publication of this Journal, but they are not owners or publishers and have no responsibility for its contents or policies.

See inside back cover or msp.org/pjm for submission instructions.

The subscription price for 2013 is US \$400/year for the electronic version, and \$485/year for print and electronic.

Subscriptions, requests for back issues and changes of subscribers address should be sent to Pacific Journal of Mathematics, P.O. Box 4163, Berkeley, CA 94704-0163, U.S.A. The Pacific Journal of Mathematics is indexed by Mathematical Reviews, Zentralblatt MATH, PASCAL CNRS Index, Referativnyi Zhurnal, Current Mathematical Publications and the Science Citation Index.

The Pacific Journal of Mathematics (ISSN 0030-8730) at the University of California, c/o Department of Mathematics, 798 Evans Hall \#3840, Berkeley, CA 94720-3840, is published twelve times a year. Periodical rate postage paid at Berkeley, CA 94704, and additional mailing offices. POSTMASTER: send address changes to Pacific Journal of Mathematics, P.O. Box 4163, Berkeley, CA 94704-0163.

PJM peer review and production are managed by EditFLOW ${ }^{\circledR}$ from Mathematical Sciences Publishers.

\section{PUBLISHED BY}

mathematical sciences publishers

nonprofit scientific publishing

http://msp.org/

(C) 2013 Mathematical Sciences Publishers 


\section{PACIFIC JOURNAL OF MATHEMATICS}

Volume $265 \quad$ No. $1 \quad$ September 2013

Genus-two Goeritz groups of lens spaces

SANGBUM CHO

A compact embedding theorem for generalized Sobolev spaces

SENG-KeE ChUa, SCOTT Rodney and Richard L. WheEden

Partial integrability of almost complex structures and the existence of

solutions for quasilinear Cauchy-Riemann equations

CHONG-KYU HAN and JONG-DO PARK

An overdetermined problem in potential theory

DMITRY KHAVINSON, ERIK LUNDBERG and RAZVAN TEODORESCU

Quasisymmetric homeomorphisms on reducible Carnot groups

XIANGDONG XIE

Capillarity and Archimedes' principle

JOHN MCCUAN and RAY TREINEN

Generalized eigenvalue problems of nonhomogeneous elliptic operators and their application

DUmitru Motreanu and Mieko TANaKa

Weighted Ricci curvature estimates for Hilbert and Funk geometries

SHIN-ICHI OHTA

On generalized weighted Hilbert matrices

EMMANUEL PREISSMANN and OLIVIER LÉVÊQUE

Unique prime decomposition results for factors coming from wreath product 221 groups

J. OWEN SizEMORE and ADAM WinCHESTER

On volume growth of gradient steady Ricci solitons

Guofang Wei and Peng Wu

Classification of moduli spaces of arrangements of nine projective lines

FEI YE 